\title{
A Novel Photon Model - Featuring an Internal Dynamics along with an Amplitude of the Oscillating Particle of Light
}

\author{
T. YARMAN ${ }^{a}$, C. YesilyURT ${ }^{a}$ AND M. ARIK ${ }^{b}$ \\ ${ }^{a}$ Okan University, Istanbul, Turkey \\ ${ }^{b}$ Boğaziçi University, Istanbul, Turkey
}

\begin{abstract}
We construct a novel model for a single photon, bearing an internal dynamics. In our model, the photon, while travelling with a speed very close to $c$ (the ultimate speed of light in empty space), is considered to oscillate, in its own frame, with its given frequency $f$. The envelope of the photon's instantaneous maximum amplitude, which we will call from here on straight "amplitude", is assumed to travel with exactly $c$, which makes that the center of the photon ought to travel with a speed little less than $c$. It is interesting to add that, in the present approach, the higher the frequency of the photon at hand, the narrower is its amplitude of oscillation. It is further assumed that the photon has a rest mass which we call "kernel", carrying the translational motion of it. This allows us to estimate the rest mass of the given photon, based on adopted initial conditions of the early universe, based on the recent Yarman and Kholmetskii model (resolving, amongst other things, the dark energy quest). One then comes out with the result that all observable photons (i.e. either high energy gamma rays or very low frequency radio waves), travel practically with the same speed, which is virtually $c$, the measured ultimate speed. A model regarding the internal dynamics in question is further offered.
\end{abstract}

DOI: $10.12693 /$ APhysPolA.125.638

PACS: 03.65. $-\mathrm{w}, 42.25 .-\mathrm{p}$

\section{Introduction}

We propose an oscillatory photon model for a single light particle. Figure 1 illustrates how we model the travelling photon, as a particle of light bearing an internal oscillatory motion; thus the photon "breathes" with a maximum amplitude "A", while it moves with a uniform translational velocity very close to $c$ (but not exactly $c$ ), in "empty space". For the time being, thus for simplicity, we assume that the photon volume (in accordance with its internal frequency), through its internal oscillatory motion, can be periodically reduced, practically to zero.

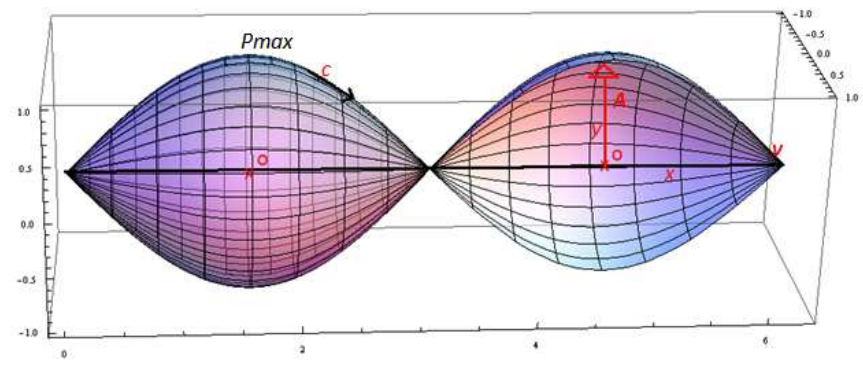

Fig. 1. Herein, the photon internal oscillation amplitude is shown, as the photon propagates along the $x$ direction. Due to the Lorentz contraction, the oscillation takes place practically in the $y z$ plane, only. The envelope drawn, by the amplitude of the oscillating particle of light picked, at a given location $x$, travels with the speed $c$, while the center $O$ of the particle of light, therefore travels with a speed $v$, little less than $c$. The $y$ direction is shown in the figure, whereas the $z$ direction is perpendicular to the plane of this page, say directed toward the reader.
The way we will elaborate on below, as the photon moves along, say, $x$ direction, its size on this direction, due to the Lorentz contraction, becomes practically zero. Hence, it can oscillate virtually on the plane $y z$, only. Thus, as the photon moves forward, along the $x$ direction, thereby, a very thin disc, practically, a circle of zero thickness, perpendicular to this direction, keeps on getting opened up, and then closed down.

Any point on the outer surface of the photon, on either possible oscillation direction, can then be viewed from the outside, as drawing a rectified sine function, as the photon moves forward, and Fig. 1 represents exactly what happens to a point on the outer surface of the kernel which is oscillating as a circle perpendicular to $x$ direction.

Below, we will consider a point on the light particle oscillating on just the $y$ direction. But what we will achieve is well valid for $z$ direction, thus as well, on any direction on the $y z$ plane.

\section{Concretization of the suggested model}

The problem which actually arises, is the following: The speed drawn by the instantaneous maximum amplitudes $\left(P_{\max }\right.$ 's) of the oscillating light particle, which we will from here on call straight "amplitude" (cf. Fig. 1) throughout the background translational motion, ought evidently to be greater than the speed of the translational motion of the center $O$ of the light particle of concern. But if the center $O$ travels with the speed $c$, the way one is usually inclined to think, then the speed drawn by $P_{\text {max }}$ 's will turn out to be greater than $c$. The special theory of relativity, though, forbids such an occurrence. Hence $O$ ought to travel with a speed less than $c$, while we can assume that the speed drawn by $P_{\text {max }}$ 's shall not exceed $c$, and in fact is exactly $c$. 
Thus, we propose to model the behavior of a point $P$ on the outer surface of the photon, oscillating along $y$ direction, with respect to $x$, as the photon moves forward, by the function

$$
y(x)=A \sin (k x),
$$

$y(x)$ is the amplitude of $P$ (which we called "instantaneous maximum amplitude" of the oscillating light particle or just "amplitude"), through the translational motion along the direction $x$, and at the location $x, A$ is the maximum amplitude, $k$ is $\frac{2 \pi}{\lambda}$, where $\lambda$ is the wavelength of the photon, i.e. more specifically the distance crossed in "empty space", by the photon, as it moves forward, while it achieves one complete internal oscillation. As proposed, we base ourselves on the fact that, the speed $c$, is the speed of the point $P$, thereby drawing the related envelope shown in Fig. 1, on the outer surface of the "light particle", through one complete internal oscillation of it, as the photon moves forward. We then, write a relationship between the photon energy $E$ and the overall envelope length $L$ of concern, of the sine function through a displacement of the "oscillating light particle" as much as as $\lambda$.

As proposed, had we assigned the speed $c$, as the speed drawn by $P$, thereby sweeping the envelope of the outer surface of the light particle, through one complete internal oscillation of it, on the way; we can throughout, write a relationship between the photon energy $E$ and the overall envelope length $L$ of concern, of the sine function through a displacement of the "oscillating light particle" as much as $\lambda$.

One can show, out of Eq. (1), that $L$ is given by

$$
L=\int_{0}^{\frac{\lambda}{2}} \sqrt{1+A^{2} k^{2} \cos ^{2}(k x)} \mathrm{d} x .
$$

Since

$$
E=h f,
$$

and by definition:

$$
c=\lambda f \text {, }
$$

we come out, with

$$
L=\frac{c h}{E}
$$

it is that, in Eq. (4), instead of the usual wavelength, we had to take into account the length $L$, to be associated with the ultimate speed $c$. In different terms, the classical definition stated by Eq. (4) must now be read as $v=\lambda f$, where $v$ is the speed of propagation of the center of the oscillating photon, while it travels, making that $v$ is just a little smaller than $c$.

Let us note that our model immediately evokes the presence of a "kernel" of rest mass $m_{0}$ of the light particle. It is, seemingly, this kernel which (i) carries the kinetic energy of the light particle, and (ii) accordingly oscillates with the usual frequency associated with the photon, which then becomes the internal oscillational frequency of the mentioned kernel, as it undergoes a uniform translational motion.

Therefore, while $P$ is moving with the speed $c$, the center of the kernel, though very close to $c$, moves with a velocity $v$, such that $v<c$.

This makes that the relativistic energy $E$ of the moving kernel is given by

$$
E=\frac{m_{0} c^{2}}{\sqrt{1-\frac{v^{2}}{c^{2}}}} .
$$

This, along with Eq. (2), yields the following relationship between $A, E$, and $\lambda$ :

$$
A=\frac{\sqrt{c^{2} h^{2}-E^{2} \lambda^{2}}}{2 E \pi} .
$$

In reality, it is not straightforward to arrive to Eq. (7) from Eq. (2), for the integration process involves an "elliptic integral". Intuitively speaking though, we can guess that the maximum amplitude $A$ is always smaller than $\lambda$, and we can guess that $A$ assumes as a maximum value, the value of $\frac{\lambda}{2}$, in which case the kernel will practically come to a rest.

Under the circumstances, one indeed goes around the elliptic integral, which becomes $\frac{\pi}{2}$, and well lands at Eq. (7).

Thus, one can write, out of Eqs. (3) and (4),

$$
\lambda=\frac{v}{c} \frac{c h}{E} \text {. }
$$

Then, Eq. (9) becomes

$$
A=\frac{c h \sqrt{1-\frac{v^{2}}{c^{2}}}}{2 \pi E},
$$

or through Eq. (6),

$$
A=\frac{h}{2 \pi m_{0} c}\left(1-\frac{v^{2}}{c^{2}}\right) .
$$

First of all, it is interesting to note that we came to something which bears the same framework as that of the uncertainty principle. Indeed Eq. (10), for $v=0$, can be written in the form of

$$
A\left(m_{0} c\right)=\frac{h}{2 \pi} \text {. }
$$

In different terms, any assumption about $A$ will yield a corresponding photon rest mass $m_{0}$. This is important for, we came to deduce that the rest mass of the photon can eventually be derived from a relationship bearing the same form as that of the Heisenberg uncertainty principle, and this is precisely that of Eq. (11).

Furthermore, Eq. (9) immediately shows that the greater $E$, the smaller is $A$, the maximum amplitude. In other words, the photon kernel travels with a speed very close to $c$, for high energies, thus much intense internal oscillation frequencies. Conversely the smaller $E$, the larger becomes the oscillation maximum amplitude.

One can anticipate to set $A$ to $\frac{\lambda}{2}$ regarding a photon which should bear the ever lowest frequency existing in the universe, and we will tackle with this interesting topic right below.

\section{Estimation of the rest mass of the photon kernel}

Based on a new cosmological model proposed by Yarman and Kholmetskii [1], the initial size of universe 
comes to be around $R_{0}=2$ billion light years. Then, there seems to be reason to assume that maximum amplitude of the photon bearing the ever longest wavelength, will assume the value of $2 R_{0}$. When this input is plugged into Eq. (8), as will be shortly shown, thus, assuming then that $\frac{v^{2}}{c^{2}}$ is consequently negligible as compared to unity, one comes out with $m_{0}$, the rest mass of the photon kernel*:

$$
m_{0}=1.6 \times 10^{-67} \mathrm{~kg} \text {. }
$$

The energy of the kernel in consideration can be expressed, as usual, as

$$
E_{0}=m_{0} c^{2} \text {. }
$$

Thus, Eq. (12) points to an energy of $1.6 \times 10^{-67} \times$ $10^{17} \mathrm{~J}=1.6 \times 10^{-50} \mathrm{~J} \simeq 10^{-21} \mathrm{eV}$. This energy is smaller by a factor of about $10^{17}$ as compared to the energy of the longest radio waves one can actually detect.

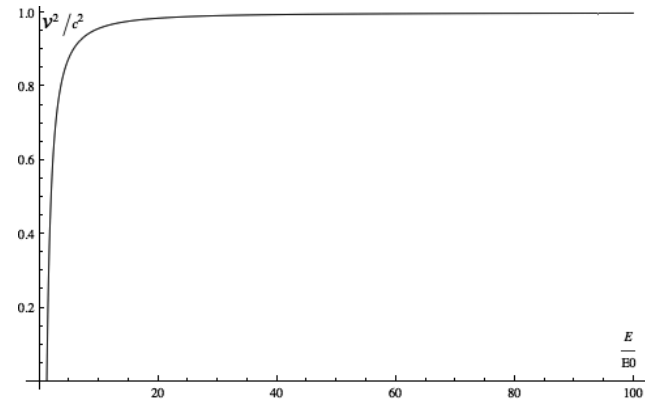

Fig. 2. The ratio of $v^{2} / c^{2}$, versus $E / E_{0}$. It is seen that for all measurable radiowaves up to high energy cosmic rays, the ratio $v / c$ is practically unity.

We can then quickly draw Fig. 2, based on Eqs. (6) and (13).

We observe that even the measurable very low frequency radio waves travel with the same speed, which is practically $c$. Thereby, it seems impossible actually to

$$
\begin{aligned}
& \text { *A more attractive way of writing Eq. (10) is } \\
& A=\frac{h}{\frac{2 \pi m_{0} c}{\sqrt{1-\frac{v^{2}}{c^{2}}}}} \sqrt{1-\frac{v^{2}}{c^{2}}}=\frac{h}{2 \pi m_{0 \gamma} c} \sqrt{1-\frac{v^{2}}{c^{2}}} \cong \frac{h}{2 \pi m_{0} \gamma c},
\end{aligned}
$$

where $m_{0 \gamma}$ is the relativistic mass of the slowest photon, though this mass is not practically any different from that expressed by Eq. (12). Yet, now going back with it, to Eq. (10), as a first iteration, will furnish the velocity of this latter photon. It turns out to be about $1 / 10$ th of $c$. This means that the rest mass $m_{0}$ is in fact little lighter than what is expressed by Eq. (10). Its energy is approximately given by Eq. (13), yielding the smallest frequency a photon can assume, and this practically at rest. When it is fully blown up, according to the present approach, it fills the entire space of the early universe. Let us note that while we came to estimate the rest mass $m_{0}$, any iteration based on it, through Eq. (10), will yield a non-zero velocity $v$, associated with it. It thus seems that the uncertainty principle, the way we landed at, forbids any absolute rest to $m_{0}$ detect in our world that, even a very low frequency radio waves travel with a speed less than $c$.

\section{Closer look at the internal dynamics of the photon}

We assumed that the photon, as a small ball, oscillates between the amplitudes 0 and $A$. Normally there are three dimensions along which it can oscillate. It seems there must be a vital Lorentz contraction along $x$ direction, i.e. that of the translational motion. There remain the two other dimensions $y$ and $z$. One can assume a Hook type of oscillation, with a restoring force $F_{y}=-k y$ and $F_{z}=-k z$; we suppose that the restoring constant $k$ is the same in both $y$ and $z$ directions.

The photon furthermore can be considered to oscillate principally in $-y$ and $y$ directions, and in $-z$ and $z$ directions, along with all the same, a maximum amplitude $A$. This makes that its internal energy $E$ can be written as

$$
E=2 \times\left(2 \times \frac{1}{2}\right) k A^{2}=2 k A^{2} .
$$

This shows indeed that the photon internal energy, for a given $k$, is proportional to the square of the maximum amplitude $A$ :

$$
A^{2}=\frac{E}{2 k} \text {. }
$$

Next, we can assume the following expression for the restoring constant $k$ :

$$
k=k_{0}\left(\frac{E}{E_{0}}\right)^{n},
$$

where $k_{0}$ is the restoring constant associated with the ever lowest frequency radio wave, of energy $E_{0}$, and $n$ is an exponent we will soon determine.

We can right away fix $k_{0}$ based on Eq. (15) and $A=R_{0}$ :

$$
k_{0}=\frac{E_{0}}{3 R_{0}^{2}} \text {. }
$$

Over here, because we deal with a photon at rest, we have taken into account the fact that the kernel oscillates principally in all three directions (and not only along $y$ and $z$ directions).

The square of Eq. (7), along with Eq. (15), leads to

$$
E^{2}=\frac{2 k c^{2} h^{2}}{4 \pi^{2}}\left(1-\frac{v^{2}}{c^{2}}\right) \text {. }
$$

This, with Eqs. (16) and (17), yields

$$
E^{3}=\frac{2 \frac{E_{0}}{3 R_{0}^{2}}\left(\frac{E}{E_{0}}\right)^{n} c^{2} h^{2}}{4 \pi^{2}}\left(1-\frac{v^{2}}{c^{2}}\right) \text {. }
$$

Over here, we propose to use Eq. (6), on the left hand side; having only constant quantities, all over, the exponent of the term $\left(1-\frac{v^{2}}{c^{2}}\right)$, must come out to be 0 . This beautifully yields

$$
n=-5 \text {. }
$$

This tells us that the greater $E$, the less intense is the restoring constant, which seems in harmony with the fact established above, as to the fact the greater $E$, the smaller is the maximum oscillation amplitude. Though the same, it is interesting to state as well that the smaller $E$, the more intense is the restoring constant. 


\section{Conclusion}

Herein we offered a photon model. Thus we assumed that the photon consists in a "light particle" bearing an internal dynamics, which oscillates with the given frequency of the photon at hand. Thus, the light particle, has a kernel, carrying the kinetic energy of the photon exhibiting a uniform translational motion. A point on the outer surface of the light particle moves with the velocity $c$, which makes that the center of the kernel should move with a velocity little less than $c$. We have shown that the greater the photon energy $E$, the smaller is the maximum amplitude of oscillation.

This allowed us to fix the rest mass of the kernel, adopting the longest wavelength of a radio wave, that may be twice of the size of the early universe [1].

We have further conjectured that the photon oscillates with its conventional frequency which led to Eq. (13); the result is interesting, stating that the conventional energy of the photon is proportional to the square of its oscillation amplitude [cf. Eq. (15)]. The proportionality constant $k$, came to be equal to $k_{0} \times\left(\frac{E}{E_{0}}\right)^{-5}, k_{0}$ being the restoring constant of the rest kernel of energy $E_{0}$ [cf. Eqs. (13), (16) and (17)]. This means that the greater $E$, the less intense is the restoring force constant. Or the same, but equally interesting, the smaller $E$, the more intense is the restoring constant.

Last but not the least, Dear Ozan Yarman, in stimulating discussions, speculated on the following alternatively valid notion, which seems to shed light as well onto the fact that the photon undergoes both a particle and a wave character. Per this outlook, the photon is hypothesized to be a Lorentz-contracted and flat-faced pressure bubble that pops in and pops out of existence, periodically, most likely, in accord with quantum uncertainty along its travel axis, on account of its frequency - resulting in the manifestation of a wave when looked at sideways (also implying a so-far neglected amplitude variable) or a blinking circle (again with an amplitude) when viewed head-on. Actually, the photon bubble - or perhaps any other elementary particle for that matter — is posited herewith to be wholly empty inside, and it is rather the surrounding medium of space that is filled, enveloping the miniscule oscillating bubble volume of practically nothingness as it propagates - much like air pockets in an adiabatic aquarium - and thereby yielding surface resistance against some sort of aether that accounts for the unbreachable ceiling velocity of $c$.

In other words, $c$ is ideally achieved only as the outermost point of the photon bubble, as it oscillates between its most outstretched maxima, and all the way down a virtually vanishing location, when it touches the minima of the wave's amplitude. Hence, the smaller the surface area of the particle bubble, the less surface resistance it encounters on its journey as it brushes against such a conjectured aether, and the more closely can its center approach the theoretical limit of the speed of light.

All this will be considered in a subsequent work. It is worth to mention that the idea in question seems capable to explain why a "photon" of a very large wavelength, thus internal oscillation amplitude can hold itself without getting ruptured down.

\section{Acknowledgments}

The authors would like to thank to Dr. Ozan Yarman, who supported the present work with long lasting discussions, thus creative ideas. One very interesting proposition he forwarded (as briefly mentioned above) consists in the fact that the perturbation, introduced as "photon" in "empty space", can be considered as a "bubble" travelling with a speed near $c$. It is worth to stress that this idea seems capable to explain why a "photon" of a very large wavelength, thus a very large internal oscillation amplitude can hold itself, as a whole entity.

\section{References}

[1] T. Yarman, A.L. Kholmetskii, Europ. Phys. J. Plus 128, 8.2 (2013). 